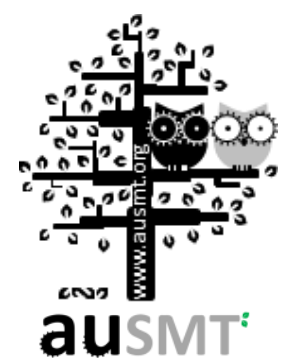

\title{
EDITORIAL
}

\section{SPECIAL ISSUE: Bio-Inspired Sensing and Actuation}

\section{Guest Editor}

\author{
Yao-Joe Joseph Yang \\ Professor/Chair \\ Department of Mechanical Engineering, National Taiwan University, Taiwan \\ yiy@ntu.edu.tw \\ DOI: $\underline{10.5875 / a u s m t . v 2 i 1.146}$
}

$\mathrm{T}$ he Earth hosts millions of living species. All of these biological systems have evolved to find sufficient solutions to survive in complex and insecure environments. Many novel and efficient engineered systems, which were inspired by adapting the principles of these solutions, have been proposed. The aim or this Special Issue of the International Journal of Automation and Smart Technology is to provide a snapshot of current research activities in the design, modeling, fabrication, characterization, and innovative applications of bio-inspired sensing and actuation systems.

Biomimetic autonomous group manipulation of mobile robots has great potential in artificial intelligence, smart life, and automation related applications. Also, it is an essential tool for exploring the behavior of biological group motion in groups such as geese and fish. In this regard, the paper entitled "Development of Navigation Schemes for Grouped Mobile Robots Leading to Biomimetic Applications" and co-authored by Ming-Hsin Chen, Yu-Te Fu, Kuang-Shun $\mathrm{Ou}$, and Kuo-Shen Chen, proposed the navigation schemes for grouped mobile robots.

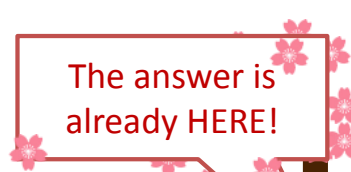

Fundamental capabilities of identification, navigation, and communication between robots were established. The tasks such as obstacle and collision avoidance, object following, autonomous movement, and the indoor localization of group robots were implemented. Essential preliminary demonstrations were performed to evaluate the effectiveness of the proposed scheme and to elucidate the potential applications of this work.

The paper entitled "Stair Climbing in a Quadruped Robot," co-authored by Shen-Chiang Chen, Chih-Chung Ko, Cheng-Hsin Li, and Pei-Chun Lin, reports the algorithm of trajectory planning and the strategy of four-leg coordination for quasi-static stair climbing in quadruped robot. This development is based on the geometrical interactions between robot legs and the stair, starting from single-leg analysis, followed by two-leg collaboration, and then four-leg coordination. In addition, the study on the quasi-static stability of the robot shows that stability can usually be maintained, and the algorithm is simulated and validated through experimentation. 
Ionic polymer metal composites (IPMCs) have been widely studied for use in biomimic transducers due to their promising biomechanical and biomimetic applications. An innovative soft tuning fork-shaped clamp transducer fabricated using IPMC is demonstrated by Guo-Hua Feng and Wei-Lun Huang, in their paper entitled "Development of Tuning Fork-shaped Clamps with Nickel-electroded lonic Polymer Metal Composites." shaped construction. Nickel was applied as the electrode material to achieve the most cost-effective device. The instance in which a sudden external force was exerted on the pinched object can also be detected from its feedback current signal.

Surface tension effect has been widely studied and employed in biomimetic devices and systems. The paper entitled "Microdroplet Protein Sensors on a Gold Surface with a Self-assembled Monolayer Treatment" by Tzu-Chun Liao and J. Andrew Yeh proposed a new kind of biological protein sensor of which the sensitivity is dominated by the surface tension of microdroplets on gold film with a self-assembled monolayer (SAM). The sensitivity of the sensor is seen to vary with the size of a microdroplet and the concentration of the detected sample (protein).

Finally, understanding biological mechanisms and integrating these mechanisms into engineered devices is promising way for developing next-generation systems of with extraordinary capacities. This research field requires the engagement of researchers from various disciplines such as biology, engineering, medical science, chemistry, materials science, and so on. In this special issue, the papers not only demonstrate the applications in different areas, but also address different aspects of bio-inspired sensing and actuating principles, and should firm up the interest in the field.
Yao-Joe Joseph Yang received his M.S. and Ph.D. degrees in electrical engineering from the Massachusetts Institute of Technology (MIT EECS) in 1997 and 1999, respectively. Also, he received his M.S. degree (1995) from UCLA and B.S. degree (1990) from NTU, both in mechanical engineering. From 1999 to 2000, he joined the Coventor Inc, (Cambridge, MA) as a senior application engineer. Since 2000, he joined the Department of Mechanical Engineering at the National Taiwan University, Taipei, Taiwan. Currently he is a professor, and serves as the chairman of the department. He is also the director for CAD Technology in the NTU NEMS Center. Since 2009, he serves as the council member of the Chinese Institute of Automation Engineering (CIAE). Currently, he is the secretary general of the Institute. Also, Dr. Yang is the council member of the ASME Taiwan Chapter.

His research interests include microelectromechanical systems (MEMS), nanotechnology, high-precision micromachining, flexible sensing arrays, sensor network, parallel processing, and semiconductor devices and vacuum microelectronics modeling. He has been consulted by more than three U.S-based companies and four Taiwan-based organizations. Dr. Yang is a member of IEEE. He is also the recipient of the Outstanding Research Award as well as Dr. Da-Yu Wu Memorial Award (Outstanding Young Research Award) of the National Science Council. 